\title{
versants
}

\section{Fotografie testi segni. Note sull'opera di Guido Guidi}

\author{
Tiziana SERENA \\ Università degli Studi di Firenze \\ ORCID: 000o-0002-988I-606I
}

\begin{abstract}
Il testo indaga la relazione fra fotografie, rappresentazioni verbali e segniche nell'opera di Guido Guidi (I94I-). Con la materia testuale e segnica, nitida e definita, offuscata e mobile, sino a divenire oggetto dell'immagine, sin dagli anni Settanta, Guidi conduce una sperimentazione linguistica sull'autonomia e l'intraducibilità del senso della fotografia.

Keywords: Guido Guidi, fotografia, materia figurativa, materia testuale, fotografia contemporanea.
\end{abstract}

Non esistono concetti a sé stanti, ma di regola sono binomi di concetti. Che cosa significa «al di sopra», mancando l'«al di sotto?» Che cosa significa «a sinistra», mancando l'«a destra»? Che cosa significa «dietro», mancando il «davanti»? [...].

I punti opposti non sono fermi, essi consentono un movimento continuo e graduale. Fermo è un punto solo, il punto centrale, nel quale i concetti sono latenti.

Paul Klee, Teoria della forma e della figurazione

Guido Guidi è, come ama definirsi, un «fotografo-fotografo»; una dichiarazione militante che sembrerebbe escludere le pratiche extralinguistiche e non puriste della fotografia. Tuttavia, sin dall'inizio degli anni Settanta, la sua materia visuale si confronta con temi e problematiche della materia testuale e segnica, principalmente tramite due tipi d'intervento. Il primo avviene ai margini del frame dell'immagine fotografica con l'inserimento di testi manoscritti, che vengono sottoposti a processi di abrasione, cancellatura, fino a diventare pressoché irriconoscibili, oppure di timbri a inchiostro con parole e numeri, segni a matita, a penna, a matita litografica, che intervengono sull'immagine, sui testi stessi e sulle cifre. Il secondo intervento è relativo a una serie dell'inizio degli anni Duemila e vede la rappresentazione verbale divenire rappresentazione visuale.

Questo saggio indaga come nella sua opera testi linguistici, parole, numeri, e segni grafici partecipino al processo creativo, stabilendo un cortocircuito fra il visivo e il verbale che costituisce un'intringante poetica della contraddizione. 
La dichiarazione della rilevanza di questa poetica è affermata dalle copertine di numerosi suoi volumi fotografici, che presentano differenti tipologie di rappresentazioni verbali e disegni a mano libera.

Il catalogo di un'importante mostra internazionale itinerante del 2014 (Guidi 20I4), presenta in copertina una fotografia che attesta il dialogo e il confronto di Guidi con la tradizione della fotografia documentaria americana. In essa è ritratto un muro di cemento su cui è stata scritta con la vernice spray la parola «Veramente», assunta a titolo del volume. Si tratta di un elemento testuale del reale trasferito in immagine che, problematizzando il tema del realismo e della veridicità della fotografia, invita lo spettatore a 'leggere' il lemma «Veramente» e a resistere alla deriva di senso che viene invocato.

Maggiormente inerente alla specificità del lavoro sulla materia testuale e segnica è il fotolibro Varianti (Guidi 1995), al cui interno Guidi ha presentato un'ampia serie di opere relazionate a segni e testi, e la serie di fotolibri recentemente edita dall'editore internazionale MACK, con la pubblicazione di lavori realizzati decenni fa. Preannunciata dalla copertina del catalogo di una mostra intitolata Guido Guidi, 1969-2004 (2004), questa serie punta sistematicamente sulla rappresentazione del valore iconico del segno verbale e grafico con le copertine di Preganziol 1983 (Guidi 2013b), Per strada (Guidi 20I8a), In Veneto (Guidi 20I9b), In Sardegna (Guidi 2019a), Lunario (Guidi 2020).

La relazione fotografie-testi-segni in Guidi è stata solo episodicamente esplorata dalla critica. Roberta Valtorta ha scritto più di altri sul tema, specificando che questa attenzione alla parola e alla scrittura verrebbe trasferita all'interno della fotografia:

La scrittura tocca la fotografia di Guidi in molti modi [...] Ma è la fotografia stessa di Guidi ad essere scrittura, o meglio progetto di scrittura, insieme di tracce per una scrittura; il colore stesso è segno che si articola e si sviluppa passando da immagine a immagine, indicando percorsi autonomi, punteggiature, direzioni (Valtorta 2003: 159).

La critica invece ha preferito analizzare temi e poetiche figurative di maggiore evidenza. Fra tutti, il binomio del valore della misurazione del rapporto spazio-tempo e il prender corpo della luce in questa misurazione, oppure l'attenzione tematica per lo spazio anodino e la sperimentazione linguistica (Dahò 20I4), la passione per i viaggi e le periferie urbane (cfr. Guidi 2003). Numerosi gli studiosi e i critici che hanno scritto sull'opera di Guidi, fra gli altri Italo Zannier, Paolo Costantini, oltre a Valtorta, a cui va aggiunto Antonello Frongia che ha esplorato il modus operandi di Guidi in un'artico- 
lata prospettiva critica e tematica in occasione di numerosi interventi (fra i quali almeno: Frongia 2013, Guidi 20I8b).

\section{2}

Attivo sin dagli anni Sessanta, Guido Guidi è salito definitivamente alla ribalta sulla scena internazionale solo in tempi abbastanza recenti. Alla fine degli anni Cinquanta approda come studente al Corso Superiore di Disegno Industriale a Venezia, dove insegnavano Luigi Veronesi, Bruno Zevi, Carlo Scarpa e, soprattutto, Italo Zannier. In seguito frequenterà la facoltà di Architettura in città. I suoi esordi 'ufficiali' nella fotografia sono legati ai risultati delle esercitazioni pratiche sul linguaggio fotografico promosse in aula da Zannier (Guidi 20I8a, in conversazione con Antonello Frongia e Andrea Simi). Grazie alla militanza del suo insegnante che promuove Guidi nelle riviste fotografiche coeve, a partire dal 1969, egli pubblica nel periodico milanese «Foto \& Film», diretto da Antonio Arcari, nell'edizione italiana di «Popular photography» e ne «Il Diaframma», entrambe edite grazie alle cure di Lanfranco Colombo (Zannier I969a, I969b, I976).

Nel corso del primo decennio della sua attività, Guidi impiega una piccola e agile macchina fotografica $24 \times 36 \mathrm{~mm}$, oltre a medi formati, e la sua riflessione si pone in confronto con le esperienze coeve artistiche d'area concettuale. Bisognerà attendere la maturazione degli anni Ottanta per vederlo utilizzare una macchina su cavalletto, un banco ottico 8xio". A partire da questa stagione e dall'impiego sistematico del grande formato, Guidi viene considerato un fotografo che potremmo sbrigativamente definire 'classico'. Classico e, allo stesso tempo, in controtendenza nelle scelte linguistiche sia nel modo di operare lento con cavalletto (all'inizio è una macchina auto-costruita in legno 50x60 cm e pesante (Guidi 20I8b: [s.p.]), sia nella scelta di stampare fotografie a contatto senza fare ricorso all'ingrandimento, mantenendo lo stesso rapporto nelle fotografie nelle pagine dei suoi fotolibri.

Nelle fotografie di Guidi, ottenute da negativo di grande formato e stampate a contatto (tranne nei casi in cui il negativo sia di formato inferiore), il rapporto di stampa I:I permette di ottenere una qualità ottica del tutto particolare. In essa si addensa una grande quantità di «minuti particolari», agglutinando quella che Daniel Arasse, nel suo magistrale volume Il dettaglio, che Guidi ha molto frequentato, ha definito una «folla di sensazioni» (2007: $\mathrm{XI})$. Dobbiamo tuttavia notare come gli stessi «minuti particolari» non coincidano esclusivamente con l'iper-realismo garantito dalla precisione ottica 
dell'apparecchiatura fotografica, ma facciano piuttosto ricorso alla retorica dello «sguardo da vicino» dello spettatore.

È lo stesso Arasse del resto a fornire questa interpretazione, ricordando le parole di Klee secondo le quali in questo avvicinamento all'opera pittorica avviene un qualcosa d'imprevisto e liberatorio, poiché la «superficie vibra» (2007: 12). Sulla superficie fotografica è spesso il dettaglio ad attirare lo spettatore, facendolo letteralmente impigliare. Ma è solo tramite l'avvicinamento che lo spettatore può fare un'esperienza particolare del dettaglio. Perché questo abbia luogo, non possiamo che supporre di trovarci di fronte a una superficie che ricorda quella che Klee aveva definito nel suo Punkt und Linie zu Fläche (I926) come «Grundfläche» e che nell'edizione italiana del I984 è stata tradotta come «superficie fondamentale»: una superficie concreta, nella sua materialità, destinata ad accogliere il contenuto dell'opera in un modo sinergico e, in quanto tale, vibrante. Il tema della vibrazione, rimanda poi alle apparenze - con le quali la fotografia sempre si misura - e a quanto ha scritto Maurice Merleau-Ponty, autore ampiamente letto da Guidi, nel testo dedicato al Dubbio di Cézanne:

Il pittore riprende e converte appunto in oggetto visibile ciò che senza di lui resta rinchiuso nella vita separata da ogni coscienza: la vibrazione delle apparenze che è la genesi delle cose (Merleau-Ponty 20ı6: 36).

La «vibrazione delle apparenze» è quanto viene trasferito nel piano della superficie fotografica, dove Guidi conduce a mio parere una sperimentazione linguistica particolare sul tema del dettaglio. Esso possiede la specifica qualità di essere antirivelatore: attira lo «sguardo da vicino», facendo avvicinare lo spettatore, e sebbene offra un supplemento visivo, la sua funzione non è quella di fornire un'aggiunta di senso, un particolare aneddotico o rivelatore, non spiega e non scioglie l'enigma della fotografia (difficile non pensare a quanto scrive Anedda 2009: 2: «Il dettaglio è isola del quadro. Per vedere meglio dobbiamo trasgredire lo spazio, abolire ogni distanza ragionevole»). Il tema del dettaglio è perlustrato da Guidi principalmente attraverso due modalità.

La prima riguarda un dettaglio visivo che agisce sulla superficie fotografica, lucida e vibrante. Qui Guidi può ricorrere a strategie che accrescono la visibilità dei dettagli visivi. Ad esempio con 'sgrammaticature' sul piano della messa a fuoco che, tramite basculaggio, risulta non parallelo al piano pellicola ma articolato lungo una diagonale, restituendo la sensazione visiva di una disarticolazione prospettica. Queste strategie figurative coinvolgono lo spettatore nella visione attraverso il riconoscimento del dettaglio che contemporaneamente, negando la propria funzione rivelatrice, non indica che il tema della visione stessa. In questo iato fra l'eccesso di vista e 
la mancanza di una denotazione e di un significato immediatamente decodificabili, Guidi individua una soglia fra il visibile (affermando la relatività delle apparenze visibili) e l'invisibile (che si manifesta attraverso esse), la cui ricerca è sempre precipua nella sua opera.

La seconda modalità è una strategia che Guidi utilizza sin dalle prime opere, negli anni Settanta, in cui lavora sul nodo fotografie-testi-segni. Essa si presenta in relazione al codice verbale e segnico, in forma di testi e linee spesso collocati ai margini del frame fotografico, ma fortemente imbricati con l'immagine e il codice figurativo. In particolare, è la materia testuale che si eleva a dettaglio nel momento in cui, come il dettaglio stesso, appare affrancata dall'imposizione di rivelare un supplemento di senso o svelare un significato. Non si tratta più, pertanto, di un dettaglio sul piano figurativo del realismo o dell'iperrealismo fotografico, realizzato al momento della ripresa, poiché la sua evidenza si manifesta nel processo successivo alla stampa anche se prende corpo sul piano della superficie fotografica.

$E$, in effetti, la superficie assume nell'opera di Guidi il valore di uno spazio tensivo, perché è qui che i valori del realismo dell'immagine, nella sua traduzione bidimensionale dello spazio tridimensionale, possono confliggere, anzi possono essere posti in conflitto dall'autore quasi con un colpo di teatro, rivelando la loro contraddizione.

\section{4}

È proprio il piano della superficie fotografica a offrirsi come una nuova tabula rasa su cui sottoporre a verifica un antico nodo, che nella sua opera si presenta come un vero e proprio «plesso concettuale», accogliendo una definizione di Andrea Pinotti per il vasto campo dei verba picta (20I8: 4I). Questo plesso è costituito dalla relazione dell'immagine fotografica con la materia testuale e grafica, in forma di parole, cifre e segni grafici apposti a matita, a inchiostro o china, o matita grassa litografica.

Nell'opera di Guido Guidi testi, parole e cifre, si presentano su due piani. Su un piano prettamente figurativo, partecipano all'immagine - come nella fotografia intitolata Senza titolo [nella copertina di Veramente] (2014) - nella forma di scritte sui muri, cartelloni stradali, brandelli di giornali e altri frammenti testuali ritrovati negli spazi pubblici. Mentre sul piano extra-figurativo troviamo una materia testuale complessa, che comprende parole, lettere, numeri e anche i segni come le linee, a cui assegna un particolare valore iconico e un potere anti-iconico. La materia testuale compare più frequentemente ai bordi del frame dell'immagine, in quella periferia dove il senso della fotografia è affidato alle parole che ne guidano la lettura o la dinegano con l'ostinazione muta delle titolazioni Senza titolo. 
Guidi in questa periferia inserisce parole e numeri, ma li sottopone a processi di abrasione che esplorano un limite-tabù: quello dell'illeggibilità del tratto, spingendosi fino alla sua pressoché totale irriconoscibilità e cancellazione. Parole e numeri vengono de-intensificati fino a renderli appena riconoscibili, quanto basta per mantenere viva la loro possibilità di erogare un senso, se non di funzionare come didascalie. Ma, di fatto, i segni linguistici si dichiarano illeggibili, non decodificabili, incapaci a comunicare il senso, o anche solo il titolo della fotografia o la sua posizione espressa in cifre nella serie. Di essi, talvolta, rimangono solo le vestigia in forma di vaghi segni fantasmatici, che sembrano invitare lo spettatore a proiettare i propri desideri e fantasie. Questo processo di disinnescamento del segno e del senso della materia testuale, che fa irruzione ai bordi dell'immagine, provoca il cortocircuito fra il visivo e il testuale. È pertanto soprattutto il lavoro sul segno linguistico che permette a Guidi di lavorare sul significato dell'immagine fotografica, d'accordo con Merleau-Ponty, per il quale «come la parola non assomiglia a quel che designa, la pittura non è un'illusione» (2016: 36).

Cancellazioni e abrasioni di parole, cifre e linee, nel momento in cui sembrano far deflagrare il loro significato, che entra in collisione con quello dell'immagine fotografica, diventano operazioni primarie in grado di mostrare il farsi e disfarsi del processo creativo e, sottolineando le fasi del processo stesso, mettono in luce il valore del tempo e il valore relativo della fotografia come immagine delle apparenze.

Nei due decenni in cui Guidi conduce maggiormente queste sperimentazioni, fra gli anni Settanta e Ottanta, la sua opera si misura con il tema della fotografia documentaria e con quello della sua veridicità anche attraverso opere meta-documentarie in cui l'oggetto è un documento vero e proprio, come un documento d'identità. Oppure il valore documentario della fotografia è affermato attraverso il ricorso a pratiche d'iscrizione che ricordano le pratiche d'archivio burocratiche, come l'uso di timbri a inchiostro recanti la data e il suo nome. Il timbro, spesso ripetuto, reso parzialmente leggibile perché trascinato o inserito fra la fotografia e la cornice, ha una funzione particolare. Soprattutto nelle serie dei primi anni Settanta, quando lo ritroviamo apposto sotto fotografie che sono state virate al tono seppia, che nella cultura visiva comune è sinonimo di antico. Anche se in questi casi si tratta di un antico artefatto, la cui funzione è quella di rimettere al centro sia il tema del tempo sia quello della veridicità dello stesso nella fotografia.

Tramite le operazioni con la materia testuale Guidi trasforma il piano bidimensionale della fotografia in una «superficie fondamentale» che si espande oltre il perimetro dell'immagine per ricomprendere e attivare il valore dei bordi. Parole e numeri si collocano «al di sopra», «al di sotto», «a sinistra» e "a destra» dell'immagine (Klee 1984: I5), nella stessa materia fotografica dove si stagliano con precisione geometrica i bordi della stessa. 
In quel bianco intonso del margine, che è ancora muta materia fotografica, dove la luce non è giunta, il segno della mano irrompe. Manifestandosi e negandosi, trasformandosi in materia iconica, introduce una sensibile effrazione di senso.

\section{5}

Nel 1995 con il fotolibro Varianti Guidi presenta al pubblico per la prima volta una retrospettiva della propria opera. In essa il plesso concettuale fotografie-testi-segni si dichiara elemento principale sin dalla copertina che, a mo' di manifesto, riproduce una fotografia dell'iscrizione manoscritta a matita del nome dell'autore e del titolo. In questo fotolibro, quasi tutte le immagini recano interventi manuali di scrittura di testi, di numeri o di segni grafici, oltre ai timbri.

Aguzzando la vista, ai margini delle immagini, cogliamo timbri, segni di linee, abrasioni, scritte a matita che presentano margini slabbrati e confusi derivanti da operazioni di cancellazione. Hanno tutta l'aria della pura accidentalità. Sembrano figure del ripensamento e della variazione. Il titolo del fotolibro, che è stato definito da Martin Parr l'equivalente italiano di American Photographs di Walker Evans (Parr-Badger 20I4: II, 80; Ragucci 20I4), omaggia una mostra omonima, Variants, organizzata da John Szarkowski al Museum of Modern Art di New York nel 1986. Ma non escluderei che il titolo rimandi a una più ampia riflessione sul tema della variatio applicata alla fotografia. Nella retorica variatio è un principio che si oppone alla iteratio, alla semplice riproduzione, e ovviamente pertiene il linguaggio, mentre nel caso di Guidi la retorica della variatio viene impiegata sia con le immagini (presentate a coppie, con lievi differenze) sia con la materia testuale e segnica (che mostra ripensamenti, cancellazioni, riquadrature), sollevando la questione del processo con cui l'immagine fotografica definisce il suo significato nel tempo.

La funzione primaria del plesso fotografie-testi-segni, difatti, mira al centro della questione verificando i limiti del significato dell'immagine fotografica. La funzione dei segni grafici e linguistici viene piegata fino alla negazione della loro stessa funzione ordinaria. I segni grafici vengono tesi fino all'incertezza della visibilità del tratto, o sperimentano la loro capacità di negazione, barrando testi, numeri e l'immagine stessa. Oppure sono linee che riconfigurano il frame fotografico, lo riducono, mostrano le possibilità di ampliamento, come nelle due fotografie sulla doppia pagina aperta in Varianti, che evidenziano il processo della scelta, del ripensamento ed esaltano lo status di provvisorietà della significazione della fotografia.

I segni linguistici vengono spinti fino al limite della loro totale illeggibilità: pur mantenendo alcuni tratti riconoscibili, si spingono sulla soglia della 
trasformazione in mero segno grafico, senza diventare totalmente tali. Si tratta di una trasmutazione, un fenomeno linguistico che volutamente non giunge a compimento risolvendosi in una forma percepita come definitiva, ma resta congelato in quella che appare come una indeterminatezza del segno. È nella forma della trasmutazione stessa che si manifesta una contraddizione poetica.

\section{6}

Nell'opera degli anni Settanta e Ottanta di Guidi le parole e i segni costituiscono pertanto coppie di poli opposti all'immagine. Punti opposti di un incessante movimento, fra immagine e testo, fra testo e segno, fra segno e immagine, fra interno figurativo ed esterno testuale, fra interno figurativo ed esterno segnico, fra comprensione e intelligibilità, come nella sperimentazione dell'alfabeto dei segni dell'esistenza di Klee e nel suo riflesso nelle esperienze di Cy Twombly. Si tratta di punti opposti che con questo movimento stabiliscono una distanza fra il frame della fotografia, che isola e blocca un frammento spazio-temporale, e la materia testuale e segnica come indice della dimensione mobile e incerta dell'uomo nel mondo.

Una distanza che intercorre fra il vecchio adagio ottocentesco di quel rapporto mimetico e quasi perfetto, sempre promesso dalla fotografia che restituisce un senso ottuso del reale, e l'abbandono trascinato del gesto della mano dell'uomo nel segno collocato ai margini della fotografia. Un gesto che parole e numeri redige, per poi confonderne il segno e denunciarne l'erosione di significato fino all'annullamento. In quel bilico che cogliamo nella trasmutazione fra segno linguistico e forma, il testo assume il ruolo di dettaglio: attira l'attenzione dello spettatore, lo compiace con la promessa di offrire un codice leggibile dell'immagine e, negando lo stesso, disattende le aspettative.

Quel «movimento continuo e graduale» di cui scrive Paul Klee (I984: I5), ruota attorno a un punto fermo e centrale. Punto che Guidi problematizza e interroga con uno sguardo ostinato sul reale. L'accenno a Klee è d'obbligo per i numerosi riferimenti fatti da Guidi sin dagli anni della formazione, forse proprio perché Klee aveva affermato e dimostrato l'autonomia dell'immagine scrivendo che la realtà dell'arte «con gli adeguati mezzi figurativi» (I984: 93) può portare «a esistenza visibile un ente che per la prima volta viene al mondo attualizzandosi in quella figura» (Spignoli 20I8: VII).

Mentre la materia testuale si fa incerta, a volte addirittura si sfalda nel segno, l'immagine sembra resistere salda e argentica, nella sua forma rettangolare o quadrata. Guidi rinuncia alla precisione del segno grafico, pur dominando la materia, lui che si è formato in ambito artistico, grafico e architettonico, o quanto meno la interroga, la mette in relazione alla precisione della fotografia per contrapporla alla veridicità.

IOO 
In tutti i casi in cui Guidi lavora con la materia testuale e segnica, dentro l'immagine, ai suoi bordi o secondo entrambe le modalità, il suo lavoro si compie sulla fotografia, smentendo la significazione primaria e culturale del significato riferibile al referente reale e alla mimesis, tramite un lavorio continuo che la ri-significa, tanto che potremmo parlare di un incessante «duplice esercizio» sulla materia testuale, segnica e figurativa, prendendo a prestito le parole che Emilio Villa aveva impiegato per l'opera del poeta visivo Luciano Caruso (Zanchetti 20I4: 66).

Le prime due fotografie, collocate dopo la pagina di guardia prima del frontespizio, presentandosi sulla doppia pagina aperta, annunciano subito la specificità del fotolibro Varianti (fig. I). Ai loro bordi vediamo segni manuali a matita, testi cancellati e un lavoro sui margini dell'immagine fotografica. Il cartoncino d'invito alla mostra presenta la fotografia della pagina destra. I dettagli che ci vengono incontro sono collocati ai margini dell'immagine e in essa agiscono, assieme al titolo della mostra e al timbro a inchiostro con il nome dell'autore che, di sbieco, invade il bordo inferiore dell'immagine.

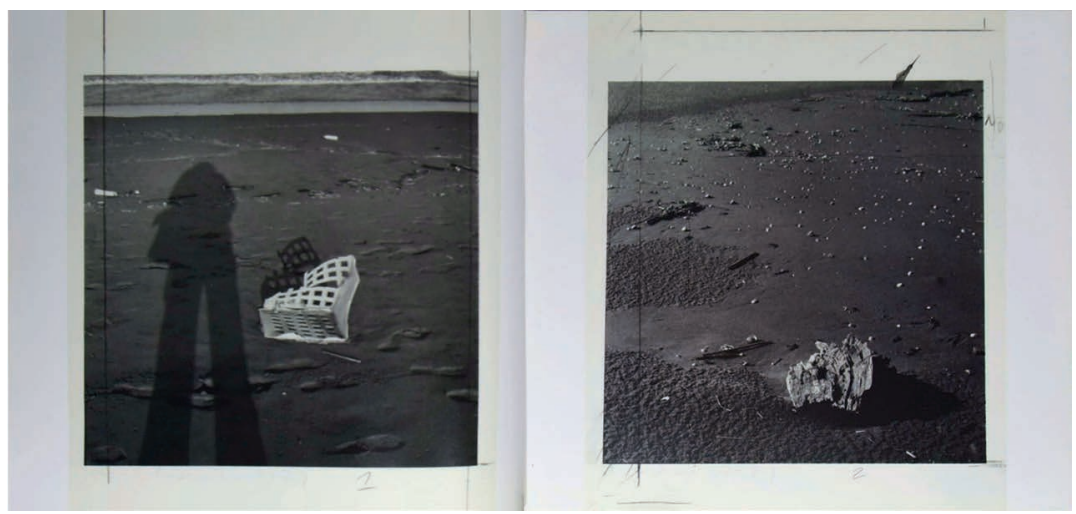

Fig. I: Guido Guidi, doppia pagina inserita prima del frontespizio di Varianti, 1994.

Ma che cosa è il margine per Guidi? Potremmo prima premettere che la questione del margine rimanda necessariamente al tema della modernità in pittura, come nel caso di Manet, il classico moderno, così come commentato da Michel Foucault, per il quale l'ékphrasis ci sposta a esplorare i bordi del quadro, fino a compierne il giro e trovare il verso (Foucault 2005: 25-46). Per Guidi, al pari, il margine rappresenta una possibilità di significare l'immagine, è una zona da attivare per fare agire la fotografia nel tempo. Essa si attiva nello spazio di un bordo per collaborare a definire i valori della 
«superficie fondamentale» (Klee I984: 195). Nelle due fotografie prima del frontespizio di Varianti, siamo di fronte a una variatio della fotografia stessa, tanto che la singola immagine viene riquadrata a matita grassa, ma sempre entro lo spazio argentico non impressionato in fase di stampa, ove risiede ancora la possibilità di essere/diventare fotografia. In questo caso Guidi inventa o solo ipotizza un orizzonte all'immagine che ne è sprovvista, che ha la capacità di elevare in potenza la forma-fotografia: il segno indica il taglio, il taglio è metafotografico, parla ancora di fotografia come gesto, come scelta.

\section{8}

In Senza titolo, 1973 («Giuliano Cosolo...») (fig. 2) dobbiamo sottolineare come, a un primo sguardo, gli apparati testuali non sembrano subito rappresentare una zona di riflessione, perché sembrano ancora assolvere alla funzione specifica informativa della didascalia. In fondo l'ambizione all'archivio, al quale la funzione della didascalia informativa demanda, appartiene alla "grande fotografia» - sostiene Guidi - come quella ottocentesca americana di Timothy O'Sullivan, che nasce proprio per essere destinata all'archivio istituzionalizzato. Tuttavia, sin dagli inizi, Guidi, pur ingaggiando la didascalia nella sua opera, dubita e fa dubitare della sua funzione primaria.

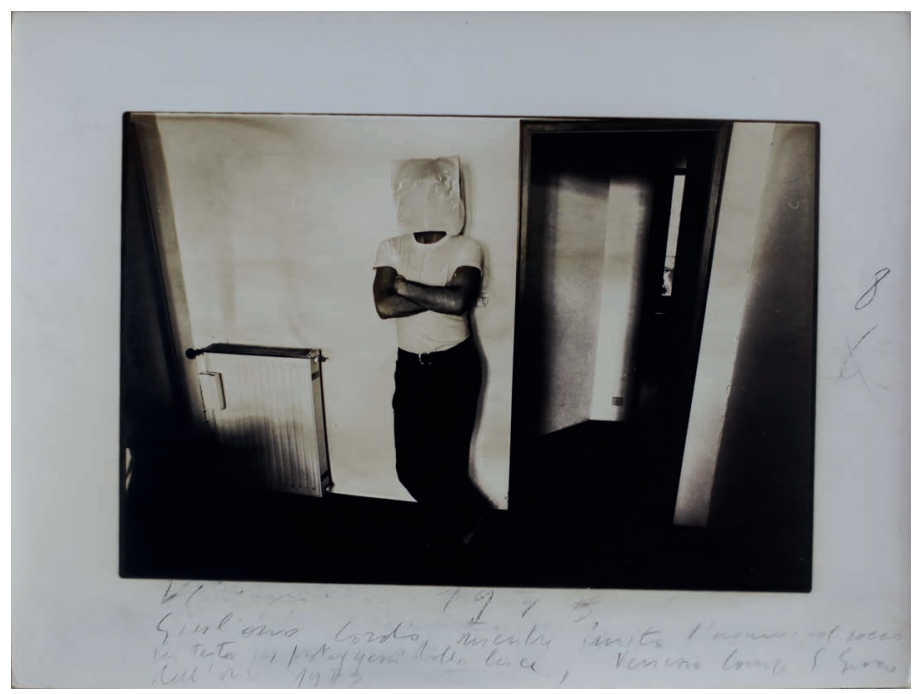

Fig. 2: Guido Guidi, Senza Titolo («Giuliano Cosolo mentre imita l’uomo con il sacco in testa proteggendosi dalla luce. Venezia Campo S. Giacomo dell’Orio, 1973»). Fotografia, 1973. (c) Guido Guidi. Cortesia dell'autore. 
In questo caso, vediamo come l'indicazione manoscritta a china e penna collocata sotto il bordo dell'immagine, risulti leggibile, anche se non rimane materia inerte. Guidi interviene su di essa e con le dita comincia a sporcare il segno linguistico, trascinandolo per ottenere irregolarità, sbavature, esplorando il limite della sua leggibilità e funzione. Giunge in questo modo a sondare il limite della soglia fra il verbale e il visivo, ottenendo un «vacillamento visivo», che ha indubbie ripercussioni sulla relazione fra lo spettatore e l'opera, e sul senso che egli vi riconosce, per certi versi analogo a quello descritto da Roland Barthes in esergo a Limpero dei segni, in cui scrive:

Il testo non "commenta" le immagini. Le immagini non "illustrano il testo": ognuna è stata per me soltanto l'inizio di un vacillamento visivo, analogo probabilmente alla perdita di sensi che lo Zen chiama un satori (1984:3).

\section{9}

A questo proposito mi pare interessante un'opera di Guidi, Senza titolo (2004), il cui incipit è «... il senso» (fig. 3). Fa parte di una seconda serie di interventi attorno al plesso fotografie-segni-testi, realizzata attorno al 2004, in cui rispetto alla serie precedente degli anni Settanta e Ottanta la rappresentazione verbale diviene rappresentazione visuale. La serie si caratterizza per l'impiego della tecnica off-camera di Man Ray, ma rimanda soprattutto ai disegni fotogenici su carta da lettera di William Henry Fox Talbot per il ricorso alla visibilità della materialità del supporto del negativo.

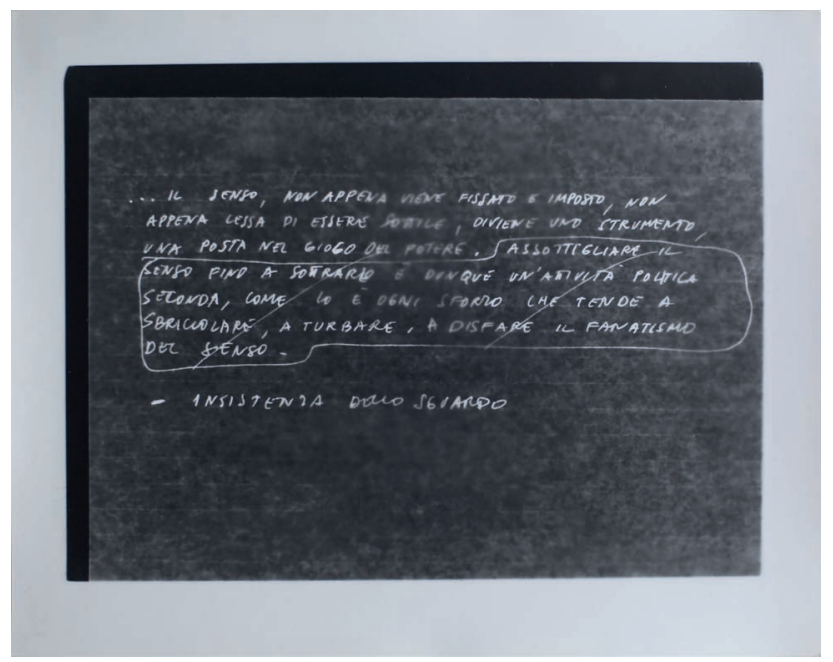

Fig. 3: Guido Guidi, Senza Titolo («Il senso...»). Fotografia, 2004. ๑ Guido Guidi. Cortesia dell'autore. 
Si tratta di una sperimentazione che Guidi compie agli inizi degli anni Duemila, riportando il discorso sulla fotografia, con la fotografia e che sembra meditare ulteriormente sulla materia testuale e segnica di Klee attraverso alcune esperienze fotografiche più contemporanee, come quella di Lee Friedlander, che fra il 1979 e il 1988 ha realizzato fotografie di graffiti, segni, testi e messaggi pubblicitari o di altro tipo, nonché cifre presenti sui luoghi pubblici, poi confluite nella mostra personale al MoMA nel 1994, Letters from the People. Non escludo anche delle suggestioni dal contesto della poesia visiva degli anni Settanta, influsso ancora da verificare.

Senza titolo (2004; «... il senso») ha come matrice negativa un foglio di carta manoscritto, la cui materia semistrasparente attraversata dalla luce crea un effetto nebuloso nero-grigio su fondo nero pece. Attorno gira un bordo bianco. Il foglio del testo non è centrato, così che in termini chiaroscurali dall'alto a sinistra passiamo dal bianco del bordo, al nero del fondo, fino al nero-grigio del foglio. Vi leggiamo:

... Il senso, non appena viene fissato e imposto, non / appena cessa di essere sottile, diviene uno strumento, / una posta nel gioco del potere.

Mentre il proseguimento sulla stessa riga del testo appare contornato da una linea e barrato, mostrando il ripensamento dell'autore che, tuttavia, non è definitivo, poiché il testo resta leggibile: il suo significato è affermato e negato allo stesso tempo, ma la significazione si mantiene come possibilità:

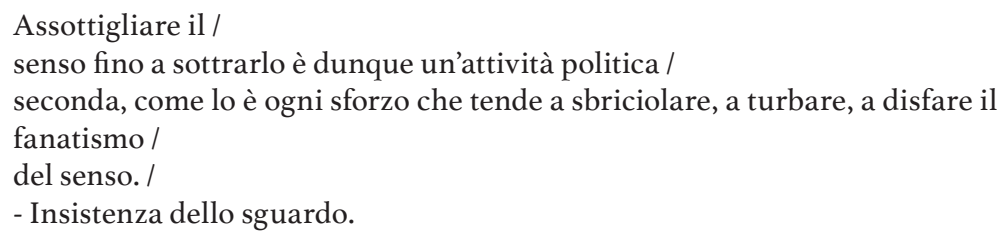

Al centro del testo-immagine «... Il senso» vi è una sfocatura. Per la grande attenzione che Guidi ha avuto per l'opera e i testi di Klee, non posso non pensare che questo suo modo di far vacillare la soglia fra rappresentazione verbale e visuale, fino a metterla (letteralmente) fuori fuoco, non abbia a che fare con la riflessione di Klee stesso sull'autosufficienza dell'immagine. In quest'opera la sfocatura è connaturata alla tecnica della fotografia off-camera, se non si utilizza volutamente un vetro per tenere uniti i due pezzi (il foglio di carta e quello fotografico, materia testuale e materia fotografica, il referente reale e la fotografia).

Quest'opera punta direttamente alla specificità del linguaggio fotografico. Intenzione, del resto, dichiarata fin dalle prime righe, dove le parole 
rimandano alla materia fotografica («fissato», "strumento») e a quella testuale, tramite il riferimento al foglio di carta («sottile»). In questo caso la cancellazione del testo non ne determina l'intelligibilità, ma anzi spinge a renderlo più evidente e la frase cassata "assottigliare ilsenso fino a sottrar= ło» sembra fare riferimento a una serie di operazioni che Guidi attua sia sul piano dell'immagine, sia sul piano della relazione con la materia testuale.

La chiusura «insistenza dello sguardo» rimanda al titolo del catalogo, L’insistenza dello sguardo. Fotografie italiane I839-I989 (I989), dove fra i co-curatori Silvio Fuso, Sandro Mescola, Italo Zannier, vi era Paolo Costantini, con il quale Guidi ha condiviso un sodalizio amicale e intellettuale, ma soprattutto alla lettera a Michelangelo Antonioni di Roland Barthes, in cui rifletteva sulla fermezza e l'insistenza dello sguardo come una contraddizione per l'artista poiché la reputava una fragilità e, allo stesso tempo, la intendeva come un supplemento d'intensità e ne sottolineava la valenza politica di sguardo radicale (Barthes 1980). Guidi sembra discutere questo passaggio proponendo come attività politica del fotografo l'assottigliamento del senso. Da questo punto di vista, la sua intenzione rimanda alle poetiche della fotografia documentaria degli anni Settanta americani, in cui la precisione descrittiva, anche attraverso il colore, nella sua valenza di senso ingombrante, poteva destabilizzare il significato dell'immagine.

Con quest'opera Guidi esplora il limite insito nel modello mimetico che per antonomasia è costituito dalla fotografia, per legarsi indissolubilmente al processo di sperimentazione del segno linguistico-figurativo nella sua irrisolutezza. Mostra la negazione delle sue funzioni informative, mette in dubbio il laccio alla referenzialità dell'uno e dell'altro linguaggio (uno alla realtà della fotografia, l'altro al referente reale), e lo riporta a livello di segno. La fotografia tutto mostra, anche come il logos si svuoti per progressioni successive, pur mantenendosi riconoscibile, sopravvivendo alle cancellazioni e al fuori fuoco.

IO

In Senza titolo (2004), incipit «Io fotografo per...» (fig. 4), con lo stesso procedimento descritto per Senza titolo (2004; incipit «... Il senso»), vediamo e leggiamo su un frammento di carta che sembra galleggiare nello spazio nero: «Io fotografo per vedere l'immagine impressa nella pellicola. Garry Winogrand». 


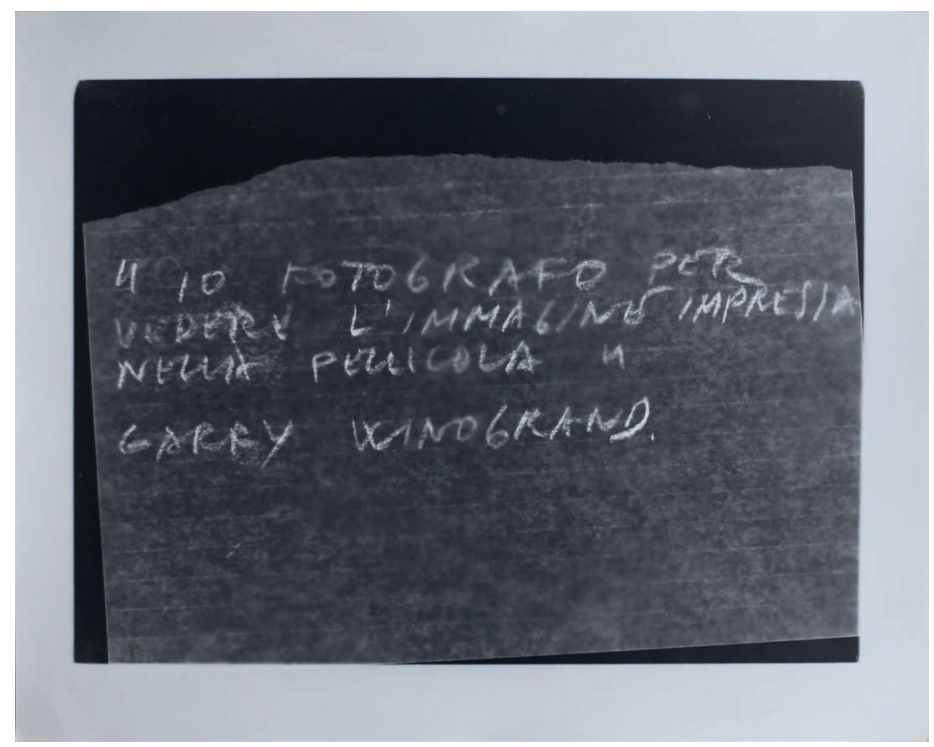

Fig. 4: Guido Guidi, Senza Titolo («Io fotografo...»). Fotografia, 2004.

(c) Guido Guidi. Cortesia dell'autore.

Guidi cita una dichiarazione celebre e dissacrante del fotografo americano, di cui ammira l'opera, realizzando una fotografia basata sul valore del frammento, pur se frammento testuale, come unità di senso isolata. Parzialità, provvisorietà, oltre ad accidentalità nella posizione sghemba del testo manoscritto, sembrano essere le valenze principali. Un'opera testuale che parla (fa leggere) della fotografia e della fotografia ripercorre il processo.

Del resto, come ha sostenuto Victor Burgin, nel suo ormai storico Looking at Photographs, il destino della fotografia è di essere sempre accompagnata da testi, di essere letta (ma non decodificata):

We rarely see a photograph in use which does not have a caption or a title [...] Even a photograph which has no actual writing on or around it is traversed by language when it is 'read' by a viewer (Burgin 1982: I44; cit. in Cometa 2016: 72).

In questa fotografia o in altre analoghe, non si tratta né di un mutuo scambio fra il verbale e il visivo, né forse di un vero incontro se non nel caso in cui Guidi presenta delle varianti alle tautologie di Joseph Kosuth, scrivendo testi che riportano le citazioni da dizionario della lingua italiana (cfr. per es. l'opera Senza titolo [2004], incipit «Facciavista...», utilizzata come quarta di copertina in Guido Guidi, 1969-2004 [Guidi 2004] con il procedimento so- 
pra descritto). Mi pare invece che un caso come quello presentato con «Io fotografo per...» possa essere trattato come un vero e proprio cortocircuito fra il visivo e il testuale che individua uno spazio, per dirla con le parole di William J. Thomas Mitchell in Picture Theory, in cui avviene una «conflagrazione fra parola e immagine» (Cammarata 2016: I6).

Siamo di fronte alla rappresentazione visuale di una rappresentazione verbale, la scrittura assume un valore iconico, o performativo come pura dichiarazione del gesto di cancellazione in cui la fotografia si confonde con il testo, diviene testo essa stessa, ma nella zona dei bordi mantiene la sua identità assoluta (bianca e nera).

Questa fotografia, Senza titolo (2004), incipit «ciò che appartiene...» (fig. 5) presenta un altro frammento. Questa volta non è il taglio del foglio di carta ad assumere le sembianze del lacerto, ma il modo con cui il testo proietta il suo segno sul foglio di carta fotosensibile.

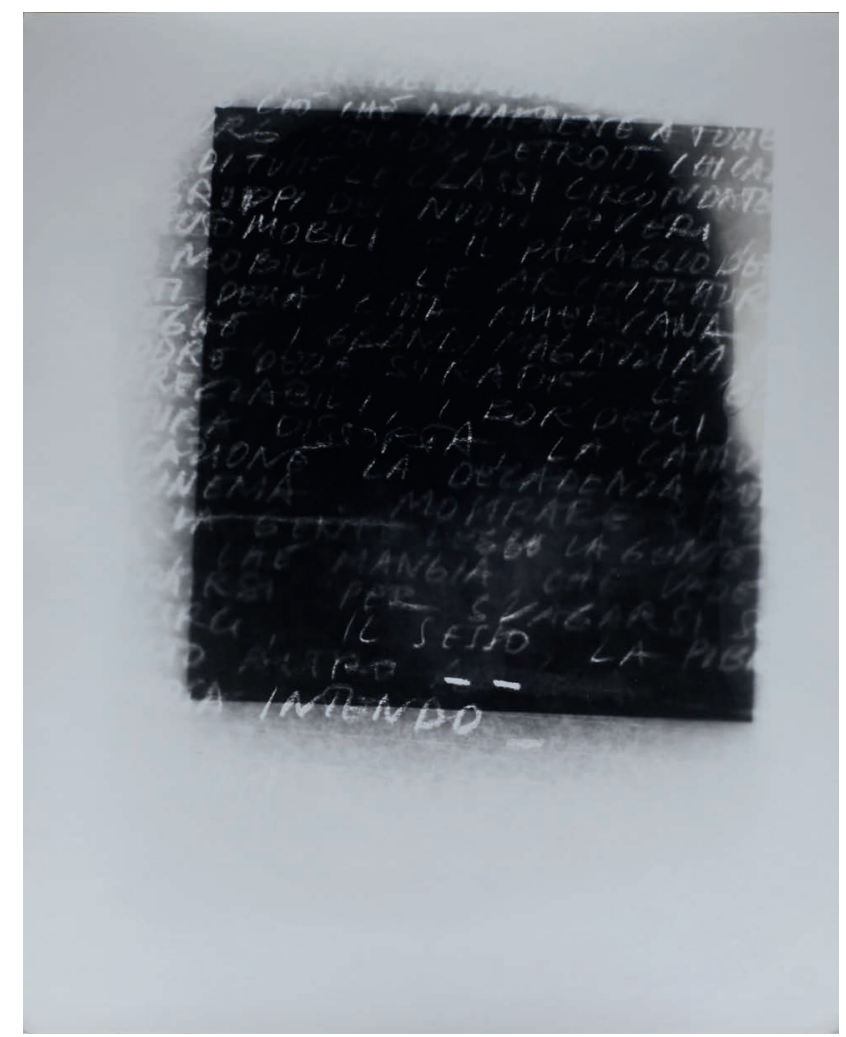

Fig. 5: Guido Guidi, Senza Titolo («ciò che appartiene...»). Fotografia, 2004.

(c) Guido Guidi. Cortesia dell'autore. 
Si tratta di un testo verbale che si sfrangia ai bordi sotto l'effetto della luce del proiettore. Nella lunga esposizione, e probabilmente grazie anche a interventi localizzati degli acidi di sviluppo che determinano una forma non omogenea, la luce si espande oltre la cornice rettangolare, assume una forma tondeggiante in cui le parole svaniscono gradualmente.

La luce informa la rappresentazione testuale, che si fa corrodere e si trasforma in foto-grafia. Allo stesso tempo, la fotografia perde la sua trasparenza nei confronti del referente reale, si fa opaca e si mostra sotto il fascio di luce. Mentre la materia testuale diviene il simbolo di una crisi del linguaggio stesso, di una impossibilità di comunicare, la fotografia ritorna in un processo di fusione, l'una nell'altra ${ }^{\mathrm{I}}$.

\section{Bibliografia}

Anedda, Antonella, La vita dei dettagli. Scomporre quadri, immaginare mondi, Roma, Donzelli, 2009.

Arasse, Daniel, Il dettaglio. La pittura vista da vicino [1996], trad. di A. Pino, Milano, Il Saggiatore, 2007.

Argan, Carlo Giulio, «Prefazione all'edizione italiana», in Klee 1984, pp. XI-XVIII.

Barthes, Roland, Limpero dei segni [1970], trad. di M. Vallora, Torino, Einaudi, 1984 .

—. «Lettre à Antonioni», Cahiers du Cinéma, 3II, I980, pp. 9-II.

Burgin, Victor, «Looking at photographs», in Id. (ed.), Thinking Photography, London, Macmillian Press LTD, 1982, pp. I42-I53.

Cammarata, Valeria, «Sfide della rappresentazione. I Trompe l'oeil di Georges Perec e Cuchi White», in Cometa-Coglitore 2016, pp. 9-48.

Cometa, Michele, «Forme e retoriche del fototesto letterario», in Cometa-Coglitore 20I6, pp. 69-II6.

Cometa, Michele - Coglitore, Roberta (a cura di), Fototesti. Letteratura e cultura visuale, Macerata, Quodlibet, 2016.

Costantini, Paolo et al. (a cura di), Linsistenza dello sguardo. Fotografie italiane I839-I989, Firenze, Alinari, 1989.

Dahò, Marta, «Through the empty room», in Guidi 20I4, [s.p.].

Donati, Riccardo, «Disobbedire all'oblio. Appunti su La vita dei dettagli», Arabeschi. Rivista di studi su letteratura e visualità, 5, 2015, pp. 15-22.

Foucault, Michel, La pittura di Manet, [2004], a cura di M. Saison, trad. di S. Paolini, Milano, Abscondita, 2005.

I Nella redazione di questo testo ho potuto discutere di alcuni passaggi con Antonello Frongia, che ringrazio. 
Frongia, Antonello, «'Tutto sotto il sole diventa vero’. Carlo Scarpa e Guido Guidi a San Vito di Altivole», in Guidi 20II, pp. 40-48.

—. «Almeno un sasso': note sulla geografia minima di Guido Guidi», in Guidi 2013a, pp. 90-95.

Guidi, Guido, Varianti, Udine, Art\&, 1995.

-. In Between Cities, a cura di M. Venturi e A. Frongia, Rubiera, Linea di Confine, 2003.

—. Guido Guidi, 1969-2004, a cura di G. Foschi, Fusignano (RA), Grafiche Morandi, 2004.

-. Carlo Scarpa's Tomba Brion, with a text by G. Guidi and an essay by A. Frongia, Ostfildern, Hatje Cantz Verlag, 20 II.

-. Cinque paesaggi, 1983-1993, a cura di A. Frongia e L. Moro, catalogo della mostra, Roma, Postcart, 2013a.

-. Preganziol 1983, [London], Mack, 2013b.

-. Veramente, catalogo della mostra (Paris, Amsterdam, Ravenna), [London], Mack, 2014.

-.Per strada, I980-I994, [London], Mack, 3 vols., 20I8a.

-. «Guido Guidi in conversazione con Antonello Frongia e Andrea Simi», in Guidi 20I8a, [s.p.].

-.In Sardegna, 1974, 20II, [London], Mack, 3 vols., 2019a.

-. In Veneto, 1984-89, [London], Mack, 2019b.

-. Lunario, I968-1999, [London], Mack, 2020.

Klee, Paul, Teoria della forma e della figurazione. Lezioni, note, saggi, raccolti ed ed. da J. Spiller, ed. it. a cura di M. Spagnol - R. Sapper, Milano, Feltrinelli, 1984 .

Merleau-Ponty, Maurice, Senso e non senso [1948], introduzione di E. Paci, trad. di P. Caruso, Milano, Il Saggiatore, 2016.

Mitchell, William J. Thomas, Picture Theory: Essays on Verbal and Visual Representation, Chicago-London, The University of Chicago Press, 1995.

Parr, Martin - Badger, Gerry (eds.), The Photobook: A History, London, Phaidon, vols. 2, 2014.

Pinotti, Andrea, «C'è proprio bisogno di dirlo? Parola e immagine dal purovisibilismo ai visual culture studies», in Spignoli 20I8, pp. 4I-43.

Pinotti, Andrea - Somaini, Antonio (a cura di), Teorie dell'immagine. Il dibattito contemporaneo, Milano, Raffaello Cortina Editore, 2009.

Ragucci, Sabrina, «Guidi. Fotografare quello che c'è», Doppiozero, 22 aprile 20I4, https://www.doppiozero.com/materiali/clic/guidi-fotografare-quello-che-c\% $3 \%$ A8 (consultato il 08.06.202I).

Spignoli, Teresa (a cura di), Verba picta. Interrelazione tra testo e immagine nel patrimonio artistico e letterario della seconda metà del Novecento, Pisa, ETS, 2018. 
Valtorta, Roberta, «La fotografia aperta di Guido Guidi», in Guidi 2003, pp. I59-I6I.

-. «Spazio, Tempo, Vuoto», in Guidi 20I3b, [s.p.].

Zanchetti, Giorgio, «Il 'duplice esercizio' di Luciano Caruso. Post-scrittura e meta-citazione», in T. Spignoli et al. (a cura di), La poesia in immagine / Limmagine in poesia. Gruppo 70. Firenze 1963-2013, Pasian di Prato, Campanotto, 20I4, pp. 65-78.

Zannier, Italo, «Esercitazioni di grammatica della fotografia. Parte seconda», Foto \& Film, 6, giugno I969a, numero monografico Il giornalismo fotografico in Italia, p. 69.

—. «Esercitazioni di grammatica della fotografia. Parte terza», Foto $\mathcal{E}$ Film, 8-9, agosto-settembre I969b, pp. I6-I7.

—. «L'avanguardia di Guido Guidi», Il Diaframma, 212, marzo 1976, numero monografico Fotografia italiana. 13 fotografi, 13 portfolii, pp. 21-25. 